Acta Bot. Croat. $71(1), 31-50,2012$

\title{
Classification of mesic grasslands and their transitions of South Transdanubia (Hungary)
}

\author{
Attila Lengyel $^{1, *}$, Dragica Purger ${ }^{2}$, JánOs CsikY ${ }^{3}$ \\ ${ }^{1}$ Department of Plant Systematics, Ecology and Theoretical Biology, \\ Eötvös Loránd University, Pázmány P. s. 1/C, H-1117 Budapest, Hungary \\ ${ }^{2}$ South Transdanubian Environment Protection Institute, Köztársaság tér 7, \\ H-7623 Pécs, Hungary \\ ${ }^{3}$ Department of Plant Taxonomy and Geobotany, University of Pécs, Ifjúság u. 6, \\ H-7624 Pécs, Hungary
}

\begin{abstract}
Relevés from meadows and pastures of South Transdanubia (Hungary) are evaluated by clustering and ordination methods. The relevé selection focused on the Arrhenatheretalia order but its transitions towards other types were also included. The groups of relevés are delimited and described according to differential, dominant and constant species. Ecological conditions of the groups were compared using indicator values. Nine groups were distinguished, four of them belonging strictly to the order Arrhenatheretalia. Each alliance of Arrhenatheretalia presented in the study area (Cynosurion, Arrhenatherion) was represented by two groups. Groups from these two alliances are separated along a light gradient, while groups of the same alliance differ in nutrient values. Within Cynosurion, the nutrient-poor group cannot be identified unambiguously as any syntaxa previously known from Hungary. The nutrient-rich Cynosurion meadows are similar to Lolio-Cynosuretum, however, they show a stronger relationship with wet meadows. Within Arrhenatherion, Pastinaco-Arrhenatheretum is recognised as a hay meadow of nutrient-rich soils. The other meadow type is similar to Filipendulo-Arrhenatheretum, thus raising syntaxonomical problems. There are transitional groups towards semi-dry and wet meadows, one dynamic phase and one outlier group among the other five clusters.
\end{abstract}

Keywords: vegetation, meadows, pastures, indicators, phytosociology, syntaxonomy

\section{Introduction}

Mesophilous grasslands are among the most species-rich herbaceous vegetation types in Hungary. Their presence is linked with the traditional agricultural system but with the intensification of farming, the area of these valuable habitats is decreasing. It is an urgent

* Corresponding author, e-mail: lengyelat@caesar.elte.hu

Copyright ${ }^{\circledR} 2012$ by Acta Botanica Croatica, the Faculty of Science, University of Zagreb. All rights reserved. 
challenge to reveal and document the diversity of mesic grasslands in order to help their conservation.

Despite their conservation importance, mesophilous meadows are a neglected category in the Hungarian vegetation classification. According to the current syntaxonomy (BORHIDI 2003), mesophilous meadows are included into the Arrhenatheretalia order. This order is represented by three alliances in Hungary: Arrhenatherion comprising lowland and colline hay meadows, Cynosurion covering lowland to montane pastures and Phyteumo-Trisetion comprising montane hay meadows; the last one is certainly not present in South Transdanubia. Previously, works aimed at synthesizing the variability of the Arrhenatheretalia order (Soó 1971, 1973; BORHIDI 2003) were based on data collected unevenly from local or subregional studies (e.g. JuHÁsz-NAgY 1959; MÁTHÉ and KovÁCs 1960; JEANPLONG 1960; KOVÁCS 1994, 2002; LÁJER 2002) without the application of more objective methods. There have been a few papers dealing with the meadows of the southern part of Transdanubia. However, they either only focus on the description of the stands of a restricted area or meadow type (DÉNES 1997, LÁJER 2002) or their sampling and analytical methodology is unclear (HoRVÁT 1962, 1972).

There are more comparative publications about other meadow types which have transitions towards mesic meadows. A classification of semi-dry grasslands has recently been prepared by ILLYÉS et al. (2009) either exclusively for Hungary, or together with other Central European countries as well (ILLYÉs et al. 2007). The classification of wet meadows of Hungary has been clarified by BOTTA-DuKÁT et al. (2005). In a few neighbouring countries, the syntaxonomy of mesophilous meadows has been investigated more thoroughly. The mesic meadows of Austria have been classified by MuCINA et al. (1993) and ElLMAUER (1994). In Slovakia, a classification has been proposed by UHLIAROVÁ et al. (2007) and JANIŠOVÁ et al. (2007) and recently refined by focusing on the Western Carpathians by RozBrojovÁ et al. (2010). In Romania meadow communities are reviewed by CoLDEA et al. (1991) and SANDA et al. (1999). Short descriptions of Croatian meadow associations are presented in TRINAJSTIĆ (2008), while recent broad-scale classification studies have been published by STANČIĆ $(2000,2008)$. There are papers containing reviews of previously surveyed meadows and pastures in Serbia (e.g. KoJIĆ et al. 2004, BLAŽENČić et al. 2005), yet they have not been evaluated nor have their results been synthesised. Some brief analyses are available for Slovenia (e.g. ČARNI 2001, ZELNIK 2007, ZELNIK and ČARNI 2008).

Our aim is to prepare the classification of the meadows belonging to the Arrhenatheretalia order in South Transdanubia and to relate our results to the existing syntaxonomical system. We intend both to differentiate among associations of this order and to delimit them from types of other orders.

\section{Materials and methods}

\section{Study area}

The study area is situated in the southern part of Hungary (Fig. 1). The major, northern part of the area belongs to the South Transdanubian geographic district, while the southern lowlands (e.g. Drava Plain) are parts of the Great Hungarian Plain (DövÉNYI 2010). For simplicity, hereafter we use the term 'South Transdanubia' for the entire study area, including its plain subregion. The southern border of the examined area is the Drava River and the 


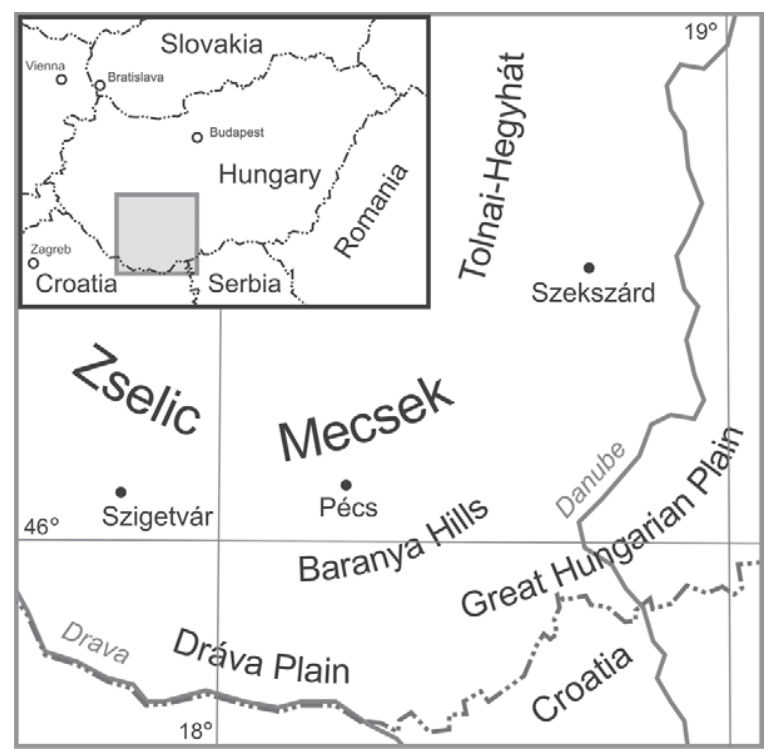

Fig. 1. The study area

national border. The other limits are defined according to the distribution of mesophilous meadows (Molnár et al. 2008), which approximately match the $700 \mathrm{~mm}$ isohyet in the east and in the north, and are delimited by the dominance of sand substratum in the west (Belsö-Somogy). At the northern part of the study range there are the Mecsek Mts, which consist of several kinds of bedrock (mainly Jurassic and Triassic limestone). This insular mountain is surrounded by hills covered by young (Pannonian, Pleistocene) sediments. South from this colline-montane landscape lies the alluvial plain of Drava River. The highest peak of the area is $682 \mathrm{~m}$ in the Mecsek Mts, while the lowest point is $87 \mathrm{~m}$ on the Drava Plain. There is a climatic continentality gradient increasing from the northwest towards the southeast (LovÁsz 1977). The study range is influenced by the sub-Mediterranean climate the most strongly in Hungary. The potential vegetation of the sites where field sampling was carried out is deciduous forest, dominated by Quercus spp., Carpinus betulus and Fagus sylvatica. Some sites located along the Drava are on drained felled alluvial forests with Quercus robur, Fraxinus angustifolia and Carpinus betulus.

\section{Vegetation data}

Field sampling was carried out from mid-May to late-June between 2004 and 2009. In total, 211 phytosociological relevés were collected according to the Zürich-Montpellier method (DENGLER et al. 2008). Plots were located in vegetation stands corresponding with the mesic meadow categories (E1, E2, E34) of the Hungarian Habitat Guide (BöLÖNI et al. 2003) which is also used for the recently finished country-wide habitat mapping project (Molnár et al. 2007). According to this system, the category of mesic meadows is somewhat wider than that of the syntaxonomic system. In terms of syntaxonomy, this allows the inclusion of Violion caninae grasslands and transitional stands towards Brometalia erecti and Molinietalia meadows, beyond Arrhenatheretalia which forms the core of this vegeta- 
tion type. During sampling, the authors preferred stands under regular and traditional management (grazing, mowing) and avoided obvious within-plot heterogeneity in factors affecting vegetation (e.g. small-scale disturbances). However, finding 'typical' stands according to the previously described associations was not aimed at since we wanted to represent the total variation as much as possible. Relevés were taken in $4 \times 4$ or $5 \times 5 \mathrm{~m}^{2}$ plots which are the recommended sizes for this vegetation type (LÁJER et al. 2008). Species cover was estimated on a percentage scale but they were log-transformed before analysis. Poorly distinguishable taxa were merged (a list is available from the authors upon request). The data set of 211 relevés was stratified geographically (KNOLLOVÁ et al. 2005) into ca. 3×3 $\mathrm{km}^{2}$ grid squares and 5 relevés were selected from each cell using the heterogeneity-constrained random resampling method (HCR, LENGYEL et al. 2011). The number of trials in HCR resampling was 10000 and the complement of the Ruzicka index (which is the Jaccard index generalized to abundance data, PODANI 2000) was used for calculating pair-wise dissimilarities between plots. The resampled data set consisted of 155 relevés.

\section{Data analysis}

Before classification, a noise elimination procedure was applied according to BOTTA-DuKÁT et al. (2005). The data set was analysed by principal coordinates analysis (applying the Ruzicka index again) and the scores of the relevés on the significant ordination axes were used as input variables for classification. The first 19 axes were considered significant because their eigenvalues exceeded the expectations based on the broken stick model (LEGENDRE and LEGENDRE 1998).

Relevé coordinates (155 relevés as objects, each with 19 scores as variables) were classified based on their pair-wise Euclidean distances using Ward's agglomerative method (PoDANi 2000). The optimal level of the clustering was determined by the Optimclass 1 method (TICHÝ et al. 2010) using $\mathrm{p}<10^{-3}$ threshold for characteristic species. Correspondingly, the dendrogram was cut at the nine-cluster level and relevé groups were described by their differential, constant and dominant species (DENGLER et al. 2008). Species with high affinity to a cluster were treated as differential species rather than truly characteristic species because in a wider comparison fidelity values may change significantly. Species fidelities to clusters were measured by the phi coefficient (CHYTRÝ et al. 2002) adjusted to equal group sizes (TICHÝ and CHYTRÝ 2006) and those reaching phi $=0.2$ and $\mathrm{p}<10^{-3}$ significance by Fisher's exact test were considered differential. Species occurring in at least $80 \%$ of the plots of a cluster were listed among the constant species. A species was considered dominant if it reached $30 \%$ cover in at least $10 \%$ of the relevés of a given cluster. Unweighted mean ecological indicator values (BORHIDI 1995, HORVÁTH et al. 1995) were calculated for each plot and clusters were compared on box-and-whisker plots.

Relationships of clusters were also examined by ordination. Canonical analysis of principal coordinates (ANDERSON and WILLIS 2003) with cluster memberships as nominal explanatory variables was applied in order to concentrate more on cluster separation. Ordination results are shown on spider plots which represent ordination and classification together. Each relevé is linked to the centroid of its group on the scatter plot, thus the diagram resembles spiders with 'legs' starting from the centroid (the 'body') and ending in the relevés' positions. Mean indicator values of relevés were passively projected onto the diagram. The goodness of fit of the projection was estimated by a permutation test. 
All analyses were performed in R Environment (version 2.10.0, R DEVELOPMENT CORE TEAM 2009), using vegan (OKSANEN et al. 2010) and cluster (MAECHLER et al. 2005) packages.

The nomenclature of plant species follows KIRÁLY (2009).

\section{Results}

\section{Description of groups}

The dendrogram of relevés is shown in figure 2. Differential, constant and dominant species are summarized in tables 1,2 and 3 respectively.

\section{Group 1}

Number of relevés: 31 ; mean species number per relevé: 41.0 .

Most relevés of Group 1 originate from moderately-grazed extensive pastures from hilltops and hillsides. In the syntaxonomic system, this type would be placed into the Cynosurion alliance within the Arrhenatheretalia order. Beyond typical elements of mesic meadows, these stands are also characterised by species tolerating nutrient-poor and dry soil (e.g. Agrostis capillaris, Anthoxanthum odoratum, Festuca pseudovina \& valesiaca, F. rubra, Hypochoeris radicata).

\section{Group 2}

This group consists of two outlier relevés, and has therefore not been interpreted.

\section{Group 3}

Number of relevés: 24; mean species number per relevé: 37.0.

Relevés classified to this group were taken in stands similar to but more disturbed than those of Group 1. The most common forms of disturbance are overgrazing, drying and soil erosion with additional leaching. Some of the stands are probably old fields which are irregularly or just recently mown or grazed. Accordingly, there are many weeds among the differential species of this group (e.g. Convolvulus arvensis, Trifolium arvense). We consider this type a dynamic state of other, more regularly managed mesic meadows, mostly of Cynosurion types.

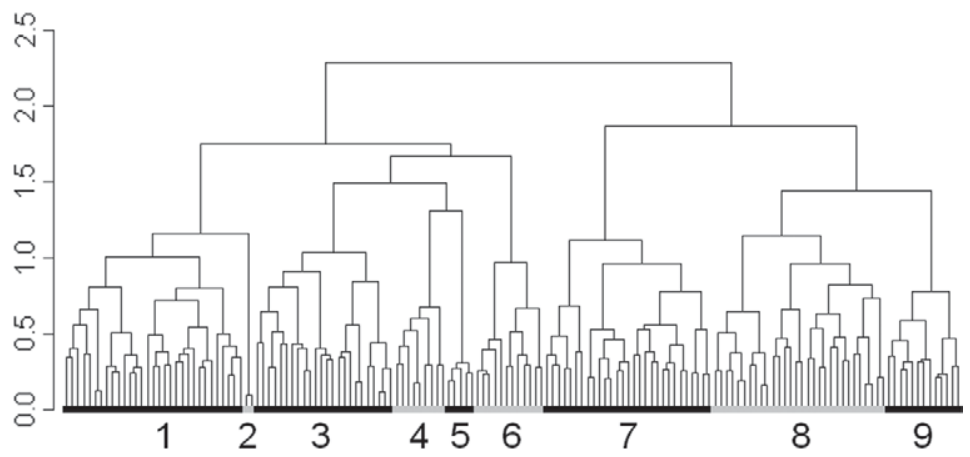

Fig. 2. Dendrogram of relevés 
Lengyel A., Purger D., Csiky J.

Tab. 1. Differential species of the groups with the values of phi coefficients.

\begin{tabular}{|c|c|c|c|c|c|c|c|c|c|}
\hline Group: & 1 & 2 & 3 & 4 & 5 & 6 & 7 & 8 & 9 \\
\hline Centaurium erythraea & 0.49 & -- & -- & -- & -- & -- & -- & -- & -- \\
\hline Leontodon autumnalis & 0.37 & -- & -- & -- & -- & -- & -- & -- & -- \\
\hline Holcus lanatus & 0.36 & -- & -- & -- & -- & -- & -- & -- & -- \\
\hline Festuca pseudov. \& valesiaca & 0.36 & -- & -- & -- & -- & -- & -- & -- & -- \\
\hline Anthoxanthum odoratum & 0.32 & -- & -- & -- & -- & -- & -- & -- & -- \\
\hline Trifolium dubium & 0.31 & -- & -- & -- & -- & -- & -- & -- & -- \\
\hline Agrostis capillaris & 0.31 & -- & -- & -- & -- & -- & -- & -- & -- \\
\hline Luzula campestris & 0.29 & -- & -- & -- & -- & -- & 0.26 & -- & -- \\
\hline Festuca rubra & 0.29 & -- & -- & -- & -- & -- & -- & -- & -- \\
\hline Daucus carota & 0.28 & -- & -- & -- & -- & -- & -- & -- & -- \\
\hline Cynosurus cristatus & 0.28 & -- & -- & -- & -- & 0.42 & -- & -- & -- \\
\hline Trisetum flavescens & 0.28 & -- & -- & -- & -- & -- & 0.27 & -- & -- \\
\hline Trifolium pratense & 0.27 & -- & -- & -- & -- & -- & -- & -- & -- \\
\hline Picris hieracioides & 0.26 & -- & 0.29 & -- & -- & -- & -- & -- & -- \\
\hline Lotus corniculatus & 0.26 & -- & -- & -- & -- & -- & -- & -- & -- \\
\hline Ranunculus bulbosus & 0.26 & -- & -- & -- & -- & -- & -- & -- & -- \\
\hline Trifolium repens & 0.25 & -- & -- & -- & -- & -- & -- & -- & -- \\
\hline Hypochoeris radicata & 0.21 & -- & -- & -- & -- & -- & -- & -- & -- \\
\hline Leontodon hispidus & 0.20 & -- & -- & -- & -- & -- & -- & -- & -- \\
\hline Centaurea stoebe & -- & 1.00 & -- & -- & -- & -- & -- & -- & -- \\
\hline Danthonia decumbens & -- & 0.98 & -- & -- & -- & -- & -- & -- & -- \\
\hline Peucedanum oreoselinum & -- & 0.95 & -- & -- & -- & -- & -- & -- & -- \\
\hline Sherardia arvensis & -- & -- & 0.55 & -- & -- & -- & -- & -- & -- \\
\hline Moenchia mantica & -- & -- & 0.49 & -- & -- & -- & -- & -- & -- \\
\hline Trifolium arvense & -- & -- & 0.48 & -- & -- & -- & -- & -- & -- \\
\hline Odontites vernus & -- & -- & 0.43 & -- & -- & -- & -- & -- & -- \\
\hline Trifolium campestre & -- & -- & 0.42 & -- & -- & -- & -- & -- & -- \\
\hline Agrimonia eupatoria & -- & -- & 0.41 & -- & -- & -- & -- & -- & -- \\
\hline Convolvulus arvensis & -- & -- & 0.39 & -- & -- & -- & -- & -- & -- \\
\hline Geranium columbinum & -- & -- & 0.38 & -- & -- & -- & -- & -- & -- \\
\hline Ranunculus polyanthemos & -- & -- & -- & 0.62 & -- & -- & -- & -- & -- \\
\hline Festuca rupicola & -- & -- & -- & 0.48 & -- & -- & -- & -- & -- \\
\hline Pimpinella saxifraga & -- & -- & -- & 0.44 & -- & -- & -- & -- & -- \\
\hline Pastinaca sativa & -- & -- & -- & 0.44 & -- & -- & -- & -- & -- \\
\hline Medicago falcata & -- & -- & -- & 0.41 & -- & -- & -- & -- & -- \\
\hline Thymus glabrescens & -- & -- & -- & -- & 0.93 & -- & -- & -- & -- \\
\hline Sanguisorba minor & -- & -- & -- & -- & 0.88 & -- & -- & -- & -- \\
\hline Brachypodium pinnatum & -- & -- & -- & -- & 0.88 & -- & -- & -- & -- \\
\hline Teucrium chamaedrys & -- & -- & -- & -- & 0.78 & -- & -- & -- & -- \\
\hline Trifolium montanum & -- & -- & -- & -- & 0.76 & -- & -- & -- & -- \\
\hline Thesium linophyllon & -- & -- & -- & -- & 0.76 & -- & -- & -- & -- \\
\hline
\end{tabular}


Tab. 1. - continued

\begin{tabular}{|c|c|c|c|c|c|c|c|c|c|}
\hline Group: & 1 & 2 & 3 & 4 & 5 & 6 & 7 & 8 & 9 \\
\hline Vicia tenuifolia & -- & -- & -- & -- & 0.76 & -- & -- & -- & -- \\
\hline Koeleria cristata & -- & -- & -- & -- & 0.76 & -- & -- & -- & -- \\
\hline Centaurea scabiosa & -- & -- & -- & -- & 0.73 & -- & -- & -- & -- \\
\hline Dianthus pontederae & -- & -- & -- & -- & 0.69 & -- & -- & -- & -- \\
\hline Asperula cynanchica & -- & -- & -- & -- & 0.68 & -- & -- & -- & -- \\
\hline Lathyrus latifolius & -- & -- & -- & -- & 0.61 & -- & -- & -- & -- \\
\hline Peucedanum cervaria & -- & -- & -- & -- & 0.61 & -- & -- & -- & -- \\
\hline Anthyllis vulneraria & - & -- & -- & -- & 0.61 & -- & - & -- & -- \\
\hline Onobrychis viciifolia & -- & -- & -- & -- & 0.61 & -- & -- & -- & -- \\
\hline Salvia pratensis & -- & -- & -- & -- & 0.61 & -- & -- & -- & -- \\
\hline Plantago media & -- & -- & -- & -- & 0.59 & -- & - & -- & -- \\
\hline Lolium perenne & -- & -- & -- & -- & -- & 0.63 & -- & -- & -- \\
\hline Agrostis stolonifera & -- & -- & -- & -- & -- & 0.62 & -- & -- & -- \\
\hline Cynodon dactylon & -- & -- & -- & -- & -- & 0.56 & -- & -- & -- \\
\hline Bromus racemosus agg. & -- & -- & -- & -- & -- & 0.50 & -- & -- & -- \\
\hline Carex distans & -- & -- & -- & -- & -- & 0.44 & -- & -- & -- \\
\hline Bellis perennis & -- & -- & -- & -- & -- & 0.43 & -- & -- & -- \\
\hline Verbena officinalis & -- & -- & -- & -- & -- & 0.42 & -- & -- & -- \\
\hline Filipendula vulgaris & -- & -- & -- & -- & -- & -- & 0.74 & -- & -- \\
\hline Betonica officinalis & -- & -- & -- & -- & -- & -- & 0.57 & -- & -- \\
\hline Carex pallescens & -- & -- & -- & -- & -- & -- & 0.49 & -- & -- \\
\hline Campanula patula & -- & -- & -- & -- & -- & -- & 0.48 & -- & -- \\
\hline Sanguisorba officinalis & -- & -- & -- & -- & -- & -- & 0.47 & -- & -- \\
\hline Vicia cracca & -- & -- & -- & -- & -- & -- & 0.40 & -- & -- \\
\hline Lathyrus pratensis & -- & -- & -- & -- & -- & -- & 0.40 & -- & -- \\
\hline Cruciata glabra & -- & -- & -- & -- & -- & -- & 0.40 & -- & -- \\
\hline Knautia drymeia & - & -- & -- & - & -- & -- & 0.40 & -- & -- \\
\hline Rumex acetosa & -- & -- & -- & -- & -- & -- & 0.38 & -- & -- \\
\hline Saxifraga bulbifera & -- & -- & -- & -- & -- & -- & 0.36 & -- & -- \\
\hline Colchicum autumnale & -- & -- & -- & -- & -- & -- & 0.31 & -- & -- \\
\hline Carex hirta & -- & -- & -- & -- & -- & -- & 0.29 & -- & -- \\
\hline Crepis biennis & -- & -- & -- & -- & -- & -- & -- & 0.28 & -- \\
\hline Ranunculus acris & -- & -- & -- & -- & -- & -- & -- & 0.26 & 0.40 \\
\hline Ranunculus repens & -- & -- & -- & -- & -- & -- & - & -- & 0.71 \\
\hline Symphytum officinale & -- & -- & -- & -- & -- & -- & -- & -- & 0.68 \\
\hline Cirsium canum & -- & -- & -- & -- & -- & -- & -- & -- & 0.64 \\
\hline Sonchus arvensis & -- & -- & -- & -- & -- & -- & -- & -- & 0.60 \\
\hline Lychnis flos-cuculi & -- & -- & -- & -- & -- & -- & -- & -- & 0.56 \\
\hline Lysimachia vulgaris & -- & -- & -- & -- & -- & -- & -- & -- & 0.47 \\
\hline Potentilla reptans & -- & -- & -- & -- & -- & -- & -- & -- & 0.46 \\
\hline Poa trivialis & -- & -- & -- & -- & -- & -- & -- & -- & 0.41 \\
\hline
\end{tabular}


Lengyel A., Purger D., CSiKy J.

Tab. 2. Constant species of the groups with percentage frequency values.

\begin{tabular}{|c|c|c|c|c|c|c|c|c|c|}
\hline Group: & 1 & 2 & 3 & 4 & 5 & 6 & 7 & 8 & 9 \\
\hline Anthoxanthum odoratum & 97 & 100 & - & -- & - & -- & 83 & - & - \\
\hline Trifolium pratense & 97 & -- & -- & -- & -- & -- & -- & -- & 85 \\
\hline Holcus lanatus & 94 & 100 & -- & -- & -- & -- & -- & -- & -- \\
\hline Lotus corniculatus & 94 & -- & -- & -- & -- & -- & -- & -- & -- \\
\hline Plantago lanceolata & 94 & 100 & 83 & 89 & -- & -- & -- & -- & -- \\
\hline Trisetum flavescens & 94 & -- & -- & -- & -- & -- & 93 & -- & - \\
\hline Achillea millefolium agg. & 90 & 100 & 88 & 100 & 80 & 92 & -- & 93 & 85 \\
\hline Galium verum & 90 & -- & 92 & 100 & - & 83 & 100 & 83 & 85 \\
\hline Poa pratensis agg. & 87 & -- & 100 & -- & 80 & 83 & 83 & 93 & 100 \\
\hline Daucus carota & 84 & -- & -- & -- & -- & -- & -- & -- & - \\
\hline Veronica chamaedrys & 81 & -- & -- & -- & -- & -- & 90 & 83 & -- \\
\hline Agrostis capillaris & -- & 100 & -- & -- & -- & -- & -- & -- & - \\
\hline Ambrosia artemisiifolia & -- & 100 & -- & -- & -- & -- & -- & -- & - \\
\hline Centaurea stoebe & -- & 100 & -- & -- & -- & -- & -- & -- & -- \\
\hline Danthonia decumbens & -- & 100 & -- & -- & -- & -- & -- & -- & -- \\
\hline Helictotrichon pubescens & -- & 100 & -- & -- & -- & -- & -- & -- & -- \\
\hline Peucedanum oreoselinum & -- & 100 & -- & -- & -- & -- & -- & -- & -- \\
\hline Festuca rupicola & -- & -- & -- & 100 & 80 & -- & -- & -- & -- \\
\hline Pimpinella saxifraga & -- & -- & -- & 89 & - & -- & -- & -- & -- \\
\hline Brachypodium pinnatum & -- & -- & -- & -- & 100 & -- & -- & -- & -- \\
\hline Plantago media & -- & -- & -- & -- & 100 & -- & -- & -- & -- \\
\hline Salvia pratensis & -- & -- & -- & -- & 100 & -- & -- & -- & - \\
\hline Thymus glabrescens & -- & -- & -- & -- & 100 & -- & -- & -- & - \\
\hline Trifolium montanum & -- & -- & -- & -- & 100 & -- & -- & -- & - \\
\hline Dianthus pontederae & -- & -- & -- & -- & 80 & - & -- & -- & - \\
\hline Sanguisorba minor & -- & -- & -- & -- & 80 & -- & -- & -- & -- \\
\hline Teucrium chamaedrys & -- & -- & -- & -- & 80 & -- & -- & -- & -- \\
\hline Alopecurus pratensis & -- & -- & -- & -- & -- & -- & 86 & 83 & 92 \\
\hline Betonica officinalis & -- & -- & -- & -- & -- & -- & 86 & -- & -- \\
\hline Lathyrus pratensis & -- & -- & -- & -- & -- & -- & 83 & -- & -- \\
\hline Ranunculus acris & -- & -- & -- & -- & -- & -- & -- & 80 & 100 \\
\hline Potentilla reptans & -- & -- & -- & -- & -- & - & -- & -- & 100 \\
\hline
\end{tabular}

\section{Group 4}

Number of relevés: 9; mean species number per relevé: 42.6.

This cluster contains transitional relevés towards semi-dry meadows, mainly from lowlands. Syntaxonomically this group should be interpreted as a cluster of transitional stands between Arrhenatheretalia and Brometalia erecti orders. Its stands are mown, grazed or overgrazed, but species-rich. Differential and dominant species are generalists and disturbance tolerants which occur both on mesic and xeromesic meadows (e.g. Festuca rupicola, Medicago falcata, Pimpinella saxifraga, Ranunculus polyanthemos). 
Tab. 3. Dominant species of the groups with percentage frequency values.

\begin{tabular}{|c|c|c|c|c|c|c|c|c|c|}
\hline Group: & 1 & 2 & 3 & 4 & 5 & 6 & 7 & 8 & 9 \\
\hline Lotus corniculatus & 23 & - & - & 11 & - & - & - & - & - \\
\hline Trifolium pratense & 19 & - & - & - & 一 & - & - & - & 23 \\
\hline Agrostis capillaris & 16 & 50 & 一 & 一 & 一 & - & - & - & 一 \\
\hline Trisetum flavescens & 16 & - & 一 & - & - & - & - & - & - \\
\hline Festuca rubra & 10 & 一 & 一 & 一 & 一 & 一 & 一 & 10 & 一 \\
\hline Holcus lanatus & 10 & - & - & - & 一 & - & 17 & 一 & 15 \\
\hline Leontodon hispidus & 10 & - & - & - & - & - & - & - & — \\
\hline Danthonia decumbens & 一 & 50 & 一 & 一 & 一 & - & 一 & 一 & 一 \\
\hline Helictotrichon pubescens & - & 50 & - & - & 一 & - & 10 & 一 & 一 \\
\hline Peucedanum oreoselinum & - & 50 & - & - & - & - & - & - & 一 \\
\hline Festuca rupicola & - & - & 25 & 22 & 40 & - & 一 & 一 & 一 \\
\hline Poa pratensis agg. & 一 & 一 & 17 & 一 & 一 & 一 & 一 & 23 & 15 \\
\hline Arrhenatherum elatius & 一 & 一 & - & 11 & - & - & - & 23 & 一 \\
\hline Brachypodium pinnatum & - & 一 & - & 11 & 40 & - & 一 & 一 & 一 \\
\hline Cynodon dactylon & 一 & 一 & 一 & 11 & 一 & - & - & 一 & 一 \\
\hline Festuca pratensis & 一 & 一 & - & 11 & 一 & 一 & - & 17 & 69 \\
\hline Medicago falcata & 一 & 一 & 一 & 11 & 一 & 一 & 一 & 一 & 一 \\
\hline Securigera varia & - & 一 & - & 11 & - & - & 一 & 一 & 一 \\
\hline Teucrium chamaedrys & - & 一 & - & - & 40 & - & 一 & 一 & - \\
\hline Bromus erectus & 一 & 一 & 一 & 一 & 20 & - & 一 & 一 & 一 \\
\hline Vicia tenuifolia & 一 & 一 & 一 & 一 & 20 & - & 一 & 一 & 一 \\
\hline Trifolium repens & 一 & 一 & - & - & 一 & 42 & 一 & 一 & 一 \\
\hline Bromus racemosus agg. & 一 & 一 & - & - & - & 17 & 一 & 一 & 一 \\
\hline Carex distans & 一 & 一 & 一 & 一 & 一 & 17 & 一 & 一 & 一 \\
\hline Alopecurus pratensis & - & 一 & - & 一 & 一 & - & 10 & 17 & 一 \\
\hline Centaurea jacea & - & - & - & 一 & 一 & - & 10 & 一 & - \\
\hline
\end{tabular}

\section{Group 5}

Number of relevés: 5; mean species number per relevé: 32.4 .

Group 5 is a semi-dry meadow group of the Brometalia order present in the Mecsek Mts. Stands are grazed or mown, and harbour far less species than the previous, transitional group. This cluster is characterised by dry grassland species (e.g. Asperula cynanchica, Dianthus pontederae, Teucrium chamaedrys, Vicia tenuifolia). The syntaxonomic position of Groups 4 and 5 should be discussed in more detail in an analysis focusing on both semi-dry and mesic meadows.

\section{Group 6}

Number of relevés: 12 ; mean species number per relevé: 30.5 .

Relevés of Group 6 originate from intensively grazed lowland pastures with nutrient-rich and well-watered soils. These plots have the lowest species numbers. The differential species of this group (e.g. Agrostis stolonifera agg., Cynosurus cristatus, Lolium 
perenne) tolerate grazing and trampling well but demand nutrient-rich and semi-humid or humid soil. Most of the sites are located on the Drava Plain where they probably originated from the overgrazing and drying of wet meadows. In the syntaxonomical system this group belongs to the Cynosurion alliance.

\section{Group 7}

Number of relevés: 29; mean number of species per relevé: 40.7.

Group 7 contains special, transitional meadows that belong to the Arrhenatherion alliance of the Arrhenatheretalia order. These stands are characterized by the co-existence of species that tolerate temporary drought and flood as well (e.g. Betonica officinalis, Filipendula vulgaris, Helictotrichon pubescens) and moisture-demanding species (e.g. Alopecurus pratensis, Carex pallescens, Sanguisorba officinalis). Besides grassland species generally characterising the mesophilous meadows (e.g. Campanula patula, Rumex acetosa, Saxifraga bulbifera, Trisetum flavescens), plants of forest edges are also present (e.g. Cruciata glabra, Knautia drymeia). This variety of species characters is explained by these meadows usually being situated on packed soils with a fluctuating water supply, because of which the moist and dry periods alternate over the year. These meadows resemble drying Molinion coeruleae stands, however, the latter is a moister grassland type. Moreover, many of these meadows are both grazed and mown, which also contributes to their diversity. These are ones of the most species-rich meadows of the region. This type is distributed in lowlands and hills.

\section{Group 8}

Number of relevés: 30; mean number of species per relevé: 34.6.

This cluster consists of relevés from regularly mown, productive, tall grass stands. These meadows grow on nutrient-rich and steadily humid soils from the lowlands to the Mecsek Mts. They are 'typical' oat grass meadows, which harbour many generalist grassland species but have very few differential species (e.g. Crepis biennis, Ranunculus acris). In the syntaxonomical system, this group corresponds to the Pastinaco-Arrhenatheretum association, which is distributed widely in other Central European countries as well. This group has transitions to almost all the others. From our preliminary analysis, we found that this group could have been delimited differently, which would slightly alter its syntaxonomic interpretation, depending on how transitional stands are classified.

\section{Group 9}

Number of relevés: 13; mean number of species per relevé: 33.

Relevés classified into Group 9 originate from the most humid sites, and should be considered as members of Molinietalia meadows. These stands are located in lowlands and valleys and they can be flooded in spring, which is not usual for mesic (Arrhenatheretalia) but typical for wet (Molinietalia) grasslands. Differential species are wet meadow plants (e.g. Cirsium canum, Poa trivialis, Ranunculus repens, Symphytum officinale). Since this group is at the periphery of the scope of our analysis, it is not discussed in more detail here.

\section{Indicator values and ordination}

Box-and-whisker plots reveal the strong outlier character of Group 5 because it considerably differs from all other groups regarding all indicator values (Figs. 3, 4, 5). Other 
groups show gradual differences in temperature (Fig. 3a), soil reaction (Fig. 4a) and continentality (Fig. $5 \mathrm{~b}$ ) values because the positions of the medians and the proportions of overlaps resemble random patterns. In contrast, groups are aggregated into 'levels' along the gradients of moisture, nutrient and light values. Groups on the same level have similar medians and highly overlapping interquartile ranges, while the levels differ significantly from each other. In moisture values (Fig. 3b), between the two extremes (Group 5 and Group 9, dry and wet, respectively) a drier level consisted of Groups 1, 3 and 4, while a moister level is formed of Groups 6, 7 and 8. There are two levels along the nutrient gradient as well (Fig. 4b). More nutrient-rich clusters are Groups 6, 8 and 9, while Groups 1, 3, 4 and 7 have on average lower nutrient status. Two levels are differentiated according to light availability values (Fig. 5a). Groups 1, 3, 4 and 6 are characterised by more light, and

a) Temperature

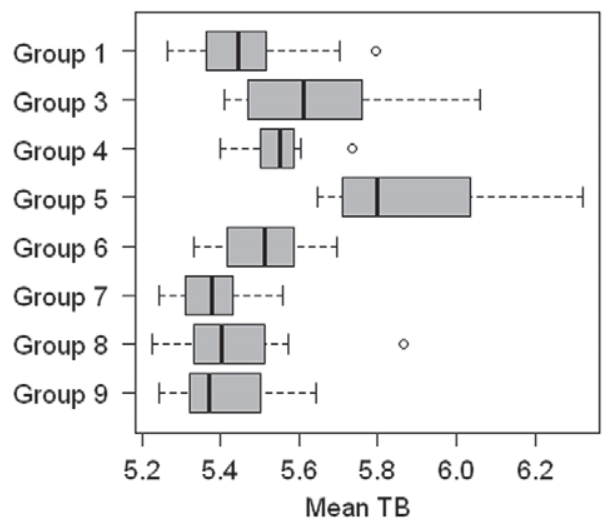

b) Moisture

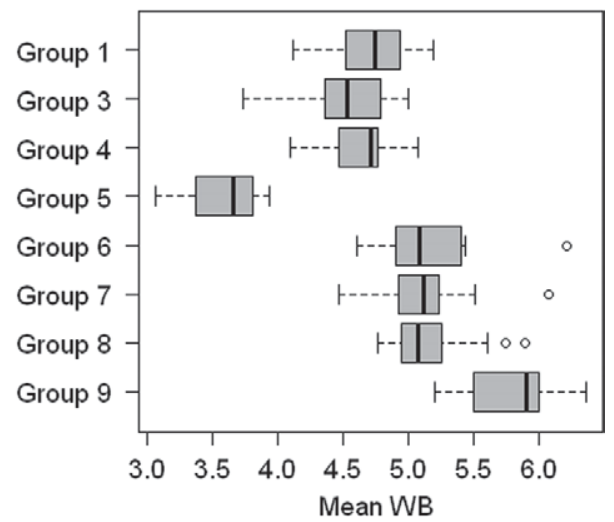

Fig. 3. Box-and-whisker plots of mean temperature (a) and moisture (b) indicator values of clusters. TB - Borhidi's temperature values, WB - Borhidi's moisture values.

a) Soil reaction

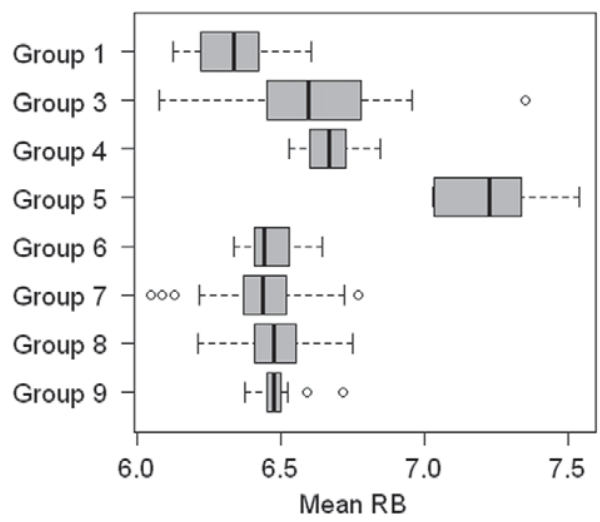

b) Nutrient status

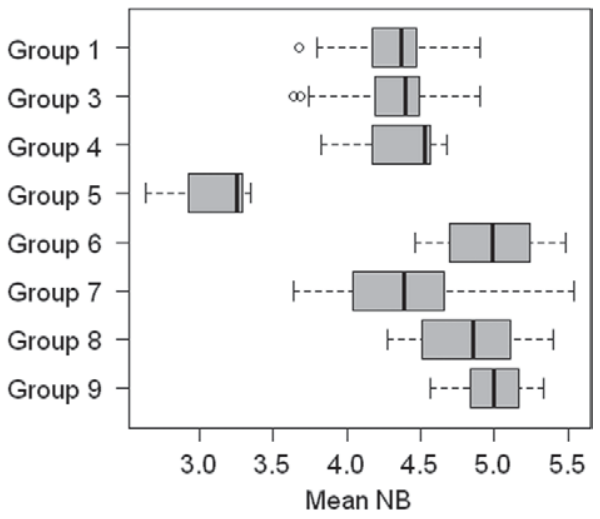

Fig. 4. Box-and-whisker plots of mean soil reaction (a) and nutrient status (b) indicator values of clusters. RB - Borhidi's soil reaction values, NB - Borhidi's nutrient demand values. 
a) Light availability

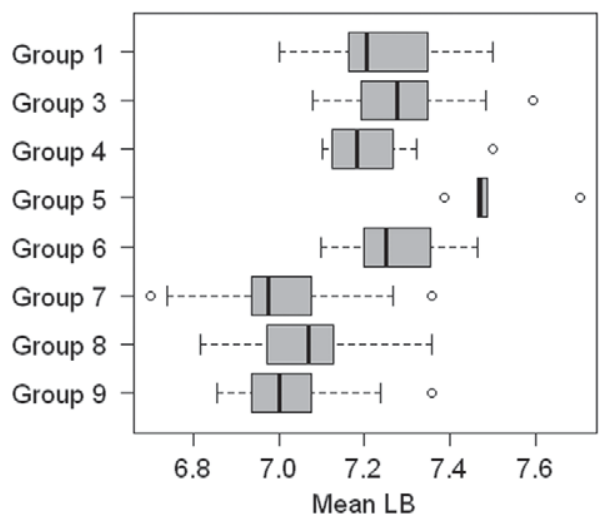

b) Continentality

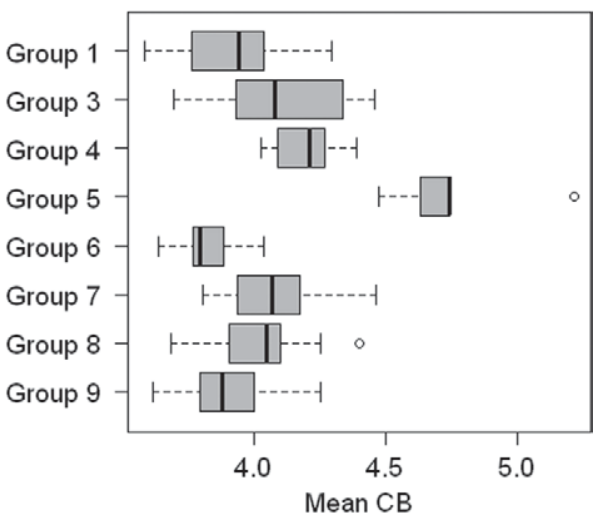

Fig. 5. Box-and-whisker plots of mean light availability (a) and continentality (b) indicator values of clusters. LB - Borhidi's light demand values, CB - Borhidi's climatic continentality

Groups 7, 8 and 9 with less. This pattern corresponds with their differences in management because relevés of Groups 1, 3, 4, 5 and 6 originate mostly from grazed sites but the others from mown meadows.

The relative positions of the groups can be seen on the ordination diagram (Fig. 6). The first canonical axis explains $23.6 \%$ of the within-group variation, which is $5.0 \%$ of the total variation. The second axis of canonical analysis of principal coordinates accounts for $20.9 \%$ of the canonical variation, which is $4.5 \%$ of the total variation of species data. Indicator val-

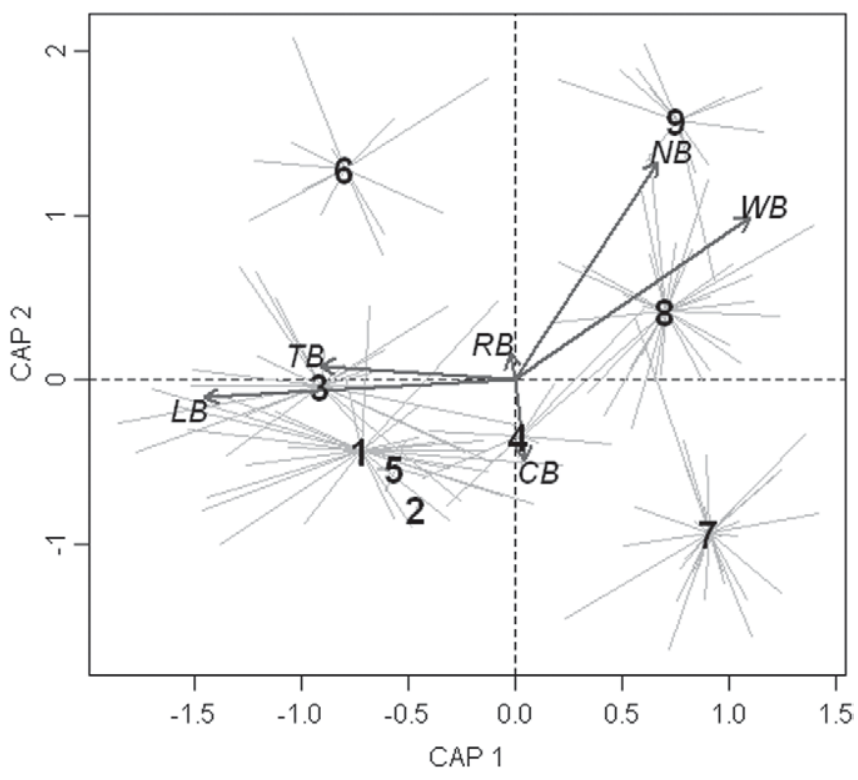

Fig. 6. Canonical analysis of principal coordinates ordination diagram with spider plot of the clusters and with passively projected mean indicator values 
ues such as: temperature indicator value, moisture, nutrient status and light availability are projected onto the scatterplot with high $(\mathrm{p}<0.001)$ continentality indicator value, with weaker but still significant $(\mathrm{p}<0.05)$ fit, while soil reaction could not be fitted to the first two axes (Tab. 4). Moisture and nutrient status correlate with both ordination axes, temperature indicator value and light availability with only the first axis, continentality indicator value with only the second axis. The ordination diagram supports the importance of moisture, nutrient status and light availability gradients in the species composition.

Tab. 4. Pearson's correlation of indicator values with the ordination axes and the goodness of fit of their passive projections. TB - temperature, $\mathrm{WB}$ - moisture, RB - soil reaction, NB - nutrient status, LB - light availability, CB - continentality indicator value

\begin{tabular}{cccccc}
\hline & \multicolumn{2}{c}{ Correlation with the axes } & & \multicolumn{2}{c}{ Fit } \\
\cline { 2 - 3 } \cline { 5 - 6 } \cline { 5 - 6 } & CAP 1 & CAP 2 & & R-squared & $p$ \\
\hline TB & -0.996 & 0.095 & & 0.209 & $<0.001$ \\
RB & 0.744 & 0.669 & & 0.544 & $<0.001$ \\
$N B$ & -0.167 & 0.986 & & 0.006 & n.s. \\
$L B$ & 0.450 & 0.893 & & 0.548 & $<0.001$ \\
$C B$ & -0.997 & -0.072 & & 0.533 & $<0.001$ \\
\hline
\end{tabular}

\section{Discussion}

Ours is the first attempt on the classification of Hungarian mesophilous meadows using multivariate methods, even if our work focuses on a minor part of the country. Therefore, our syntaxonomic results generate rather new questions and hypotheses about the current syntaxonomic system. From our classification four clusters emerged as important types certainly belonging to the Arrhenatheretalia order.

Two of these four clusters belong to the Cynosurion alliance (Group 1, Group 6) and the other two to Arrhenatherion (Group 7, Group 8). These alliances are separated along the first axis of the canonical principal coordinate ordination that corresponds also with light availability (Fig. 6), with groups of Cynosurion getting higher mean light availability values. This is in line with the differences in their management (i.e. grazing for Cynosurion and mowing for Arrhenatherion), and accordingly Borhidi's light values seem to differentiate well between grazed and mown communities.

Within both alliances, clusters differ in nutrient status (Figs. 4b, 6). These patterns were more or less incorporated previously in the syntaxonomic concept but species compositions of the groups were not in accordance with the association descriptions in every case. In the Hungarian syntaxonomic system (BORHIDI 2003), mesophilous pastures on nutrient-poor soils are included in the Festuco commutatae-Cynosuretum distributed in colline and montane areas, which contains species associated with the montane climate in Hungary (Carlina acaulis, Rhinanthus alectorolophus, Rh. wagneri, Danthonia decumbens, Alchemilla spp.). However, even the highest altitudes of our study area do not reach the montane belt and most of the montane characteristic species of the above association are not present in the regional species pool. Thus, pastures with low nutrient availability in 
South Transdanubia did not correspond with Festuco commutatae-Cynosuretum; however, this can be explained by the lack of data from this region in the past, which prevented the Hungarian description (BORHIDI 2003) of this association being extended to the southern stands. As borne out by RozBrojová et al. (2010), extensive pastures have species compositions similar to low production hayfields. There is a low productive hay meadow association in Hungary in the Arrhenatherion alliance, Anthyllido-Festucetum rubrae, which is distributed through the whole country in colline and montane ranges according to BORHIDI (2003). However, we could not recognise this association based on our data. It seems time to revise these two association names and descriptions (Festuco commutatae-Cynosuretum and Anthyllido-Festucetum rubrae) based on statistical analyses, limit their application to narrower geographic areas and find appropriate names for the others. Moreover, if uniqueness and distinctness of narrow-range associations are not supported enough, it is advisable to adopt names from the syntaxonomic systems of neighbouring countries in order to standardise the nomenclature of syntaxa over the countries (KNOLLOVÁ et al. 2006). Based on archive data and available literature of Central European countries (HÁJKovÁ et al. 2007, JAnISOVÁ et al. 2007), Festuco-Cynosuretum, Anthoxantho-Agrostietum and Festuco-Agrostietum seem equally applicable names for southern Hungarian extensive pastures.

The group of nutrient-rich Cynosurion pastures (Group 6) resembles the Lolio-Cynosuretum association that is already reported from Hungary. According to BoRHIDI (2003) this association occurs in colline and montane ranges. However, HÁJKOVÁ et al. (2007) extended its altitude range to the lowlands in the Czech Republic. In our study area, similar stands are the most common in the Drava Plain, but rarer in the higher altitudes, contrary to BORHIDI (2003). Lowland stands are not only situated at lower altitudes but also in southern geographic position affected more by sub-Mediterranean climate and they harbour a few of the sub-Mediterranean species that are rare or sparse in the study region (e.g. Hordeum secalinum). Therefore, we think an urgent task to explore the relationship between the nutrient-rich Cynosurion pastures of South Transdanubia and the Bromo-Cynosuretum stands of Croatia. It should also be further investigated how they are delimited from trampled and grazed wet meadows. This problem is also recognised by ZuIDHOFF et al. (1995) who suggested applying the name Junco-Cynosuretum Sougnez 1957 to relevés that they classified to a transitional cluster between Calthion and Cynosurion (mainly Lolio-Cynosuretum). Noteworthily, their analysis is based mostly on Western European relevés with disproportionately few Central European data.

We identified Group 8 as a Pastinaco-Arrhenatheretum elatioris association that is a common meadow community in Central Europe and also included in the Hungarian system. Nevertheless, there are some other associations characterised by Arrhenatherum elatius which are difficult to distinguish from Pastinaco-Arrhenatheretum. In Hungary, such an association is Alopecuro-Arrhenatheretum which is a mountainous type described from the northern part of the country (MÁthé and KovÁcs 1960). In Croatia, Ononido-Arrhenatheretum is distributed along the Drava and Mura rivers and is considered a moister and less productive meadow than Pastinaco-Arrhenatheretum (ILIJANIĆ and ŠEGULJA 1983, TRINAJSTIĆ 2008). Though, these associations are not clearly delimited from Pastinaco-Arrhenatheretum (STANČIĆ 2008) and the differences are probably so slight that the applied hierarchical classification methods are not able to distinguish them as separate clusters of our current data set. Here we suggest defining these associations by supervised 
classification tools (BRUELHEIDE 2000) and/or calculating similarity measures (TICHÝ 2005, VAN TONGEREN et al. 2008) between reliable reference plot data and the unidentified relevés, and using the resulting assignments for mapping their distribution. We expect that a few relevés would be identified as any of these rarer associations but the majority of Group 8 should be assigned to Pastinaco-Arrhenatheretum. It is also worth mentioning that Pastinaco-Arrhenatheretum is characterised by generalist meadow species, which are common on drying wet meadows and other dynamic types, thus the species composition of Pastinaco-Arrhenatheretum can easily appear on various sites. It is another question whether differences among the above narrow associations (Alopecuro-Arrhenatheretum and Ononido-Arrhenatheretum) and the core type (Pastinaco-Arrhenatheretum) are bigger than the differences among dynamic phases.

The low productive part of Arrhenatherion alliance had previously been covered by two associations: Anthoxantho-Festucetum rupicolae from lowlands and Anthyllido-Festucetum rubrae from colline and montane ranges (BORHIDI 2003). Anthoxantho-Festucetum rupicolae is originally described from this area (Drava Plain) by DÉNES (1997) and some of our relevés are clearly identifiable with the published data. Anthyllido-Festucetum rubrae is considered common in deciduous forest ranges (BORHIDI 2003), but we could not recognise it in our data. Instead, our Group 7 seemed the most similar to Filipendulo-Arrhenatheretum described originally from Austria (HUNDT and HüBL 1983) and detected also in Croatia (TRINAJSTIĆ 2002). Similar stands are reported from the Bakonyalja region in Transdanubia (BAUER et al. 2001), but no phytosociological relevés are published. The presence of this association in Hungary should be validated by analysing our data together with relevés of the above countries. If this community is accepted as present in Hungary, the position of Anthoxantho-Festucetum rupicolae should also be revised because Filipendulo-Arrhenatheretum would be an association with a wide distribution and many variants, and Anthoxantho-Festucetum rupicolae may be only its dry variant with subregional distribution. In Austria three subassociations of Filipendulo-Arrhenatheretum are described in HUNDT and HüBL (1983): typical, wet (with Cirsium oleraceum) and dry (with Centaurea scabiosa), although the dry subassociation differs from Anthoxantho-Festucetum rupicolae floristically. Recently, an ecologically similar association was described (ZELNIK 2007) with the name Triseto-Centaureetum macroptili. This community represents transitions between wet and mesic grasslands and it occurs on elevated part of alluviae where the water table fluctuates, as in the locations of Group 7. However, we are not aware of any occurrences of its dominant species, Centaurea jacea subsp. macroptilon, in the studied region. Moreover, Triseto-Centaureetum harbour some acidophilous species that are also very rare or absent in South Transdanubia (e.g. Nardus stricta, Festuca filiformis).

We also identified a dynamic phase of several types but mainly of nutrient-poor pastures. Such clusters are usually not interpreted syntaxonomically. However, these degraded stands show continuous transition to other, more 'stable' types and their representation in an unsupervised classification is also a question of sampling decisions.

Three of our clusters are identified as belonging to or representing transitions to orders other than Arrhenatheretalia. Aware that the transitions between Arrhenatheretalia and Brometalia or Arrhenatheretalia and Molinietalia meadows are continuous and they can only be delimited quite arbitrarily, we are careful with the syntaxonomic interpretation of 
transitional groups. These clusters are hard to characterise because they often include stands from various transitional pathways between broader syntaxa. For example, Group 4 comprises a broad variety of transitions between mesic and semi-dry meadows, its diagnostic species thus being the generalists, which have in common only their moisture preference. Heterogeneity of this group is also supported by Festuca rupicola being the only species that dominates more than one stand. There are three associations considered transitional between semi-dry and mesic meadows by BoRHIDI (2003): Anthoxantho-Festucetum rupicolae Dénes 1997 in the Arrhenatherion alliance, Onobrychido viciaefoliae-Brometum erecti Müller 1966 and Carlino acaulis-Brometum Oberdorfer 1957 in the Bromion erecti, however, the presence of the latter two is not validated by statistical analysis in a recent review (ILLYÉs et al. 2009). Literatures of neighbouring countries also consider Ranunculo bulbosi-Arrhenatheretum Ellmauer in Mucina et al. 1993 and Filipendulo vulgaris-Arrhenatheretum Hundt et Hübl 1983 of Arrhenatherion as such intermediate types. There are several associations interpretable as transitions between wet and mesic meadows as well (e.g. Alopecuro-Arrhenatheretum (Máthé et Kovács 1960) Soó 1971, Cirsio cani-Festucetum pratensis Májovsky et Ružičková 1975, Arrhenathero-Molinietum arundinaceae Lájer 2002, Holcetum lanati Issler 1934). In our opinion, the status of these intermediate associations should be investigated in an analysis comprising many relevés from both orders they are related to.

\section{Acknowledgements}

We are grateful to János BoDÓ, András PinTÉr, István Zsolt TóTH and László WÁGNeR for their help in the field work. We thank Prof. János PODANI and the two anonymous referees for their valuable comments on the manuscript.

\section{References}

Anderson, M. J., Willis, T. J., 2003: Canonical analysis of principal coordinates: a useful method of constrained ordination for ecology. Ecology 84, 511-526.

Bauer, N., BAlogh, L., KenYeres, Z., 2001: Vegetation and conservational problems of the fens at Tapolcafö and Attya (Pápa-Bakonyalja; Region of the Bakony Mountains, Hungary) (in Hungarian). Botanikai Közlemények 88, 71-94.

BlažEnčić, J., Ranđelović, V., Butorac, B., Vukojičić, S., Zlatković, B., Žukovec, D., ĆAlić, I., PAVIĆEvić, D., LAKUŠıć, D., 2005: Habitat classification of Serbia (In Serbian). Institut za Botaniku i Botanička Bašta Jevremovac, Belgrade.

BölÖNI, J., Kun, A., MolnáR, Z. s., 2003: Habitat guide (In Hungarian). MTA ÖBKI, Vácrátót.

BoRHIDI, A., 1995: Social behaviour types, the naturalness and relative ecological indicator values of the higher plants in the Hungarian flora. Acta Botanica Hungarica 39, 97-181.

BorHIDI, A., 2003: Plant associations of Hungary (In Hungarian). Akadémiai Kiadó, Budapest.

Botta-Dukát, Z., Chytrý, M., Hásková, P., Havlová, M., 2005: Vegetation of lowland wet meadows along a climatic continentality gradient in Central Europe. Preslia 77, 89-111. 
BRuelHeide, H., 2000: A new measure of fidelity and its application to defining species groups. Journal of Vegetation Science 11, 167-178.

ČARNI, A., 2001: Vegetation of cultivated grasslands in the Goričko region (NE Slovenia). Acta Biologica Slovenica 44, 13-27.

Chytrý, M., TichÝ, L., Holt, J., BotTA-DukÁt, Z., 2002: Determination of diagnostic species with statistical fidelity measures. Journal of Vegetation Science 13, 79-90.

ColdeA, G., 1991: Prodrome des associations vegetales des Carpates du sud-est (Carpates Roumaines). Documenta Phytosociologica 13, 317-539.

DÉNES, A., 1997: Occurrence of Anthoxantho-Festucetum rupicolae association on the meadows of the Drava Plain (In Hungarian). Kitaibelia 2, 274-275.

Dengler, J., Chytrý, M., Ewald, J., 2008: Phytosociology. In: Jörgensen, S. E., Fath, B. D., Encyclopedia of ecology 4. General ecology. Elsevier, Oxford.

DövÉNYI, Z., 2010: Magyaroszág kistájainak katasztere. MTA Földrajztudományi Kutatóintézet, Budapest.

Ellmauer, T., 1994: Syntaxonomie der Frischwiesen (Molinio-Arrhenatheretea p.p.) in Österreich. Tuexenia 14, 151-168.

Hájková, P., Hájek, M., Blažková, D., Kučera, T., Chytrý, M., ØezníčKová, M., Šumberová, K., Černy, T., Novák, J., Simonová, D., 2007: Meadows and mesic pastures. In: ChytrÝ, M. (ed.), Vegetation of the Czech Republic. 1. Grassland and heathland vegetation (In Czech). Academia, Praha.

Horvát, A. O., 1962: Meadows in the vicinity of the Mecsek Mts. (In Hungarian) Annals of the Janus Pannonius Museum 1960, 53-67.

Horvát, A. O., 1972. Die Vegetation des Mecsekgebirges und seiner Umgebung. Akadémiai Kiadó, Budapest.

Horváth, F., Dobolyi, Z. K., MorschHauser, T., Lõkös, L., Karas, L., Szerdahelyi, T., 1995: Hungarian flora database 1.2. List of taxa and relevant attributes (In Hungarian). MTA ÖBKI, Vácrátót.

Hundt, R., HÜBL, E., 1983: Pflanzensoziologische, pflanzengeographische und landeskulturelle Aspekte des Filipendulo-Arrhenatheretum im Wiener Wald. Tuexenia 3, 331-342.

ILIJANIĆ, Lj., ŠEGUlJA, N., 1983: Phytozönologische und ökologische Untersuchungen der Glatthaferwiesen in der Podravina (Nordkroatien). Acta Botanica Croatica 42, 63-82.

Illyés, E., BAuer, N., BotTA-Dukát, Z., 2009: Classification of semi-dry grassland vegetation in Hungary. Preslia 81, 239-260.

Illyés, E., Chytrý, M., Botta-DukÁt, Z., Jandt, U., ŠKodovÁ, I., JanišovÁ, M., WillNER, W., HÁJEK, O., 2007: Semi-dry grasslands along a climatic gradient across Central Europe: Vegetation classification with validation. Journal of Vegetation Science 18, 835-846.

Janišová, M., Michálková, D., Škodová, I., Uhliarová, E., Zaliberová, M., 2007: Cynosurion cristati Tüxen 1947. In: JANIŠOvÁ, M. (ed.), Grassland vegetation of Slovak Republic: electronic expert system for identification of syntaxa (In Slovakian), 110-118. Botanický ústav SAV, Bratislava. 
JEANPlONG, J., 1960: Übersichliche Schilderung der am oberen Raablauf in Ungarn gelegenen Wiesen (In Hungarian). Botanikai Közlemények 48, 101-105.

JuhÁsZ-Nagy, P., 1959: Meadow and pasture associations of the Bereg Plain (In Hungarian). Acta Universitatis Debreceniensis 4, 195-228.

KIRÁLY, G. (ed.), 2009: New Hungarian herbal. The vascular plants of Hungary. Identification key (In Hungarian). Aggteleki Nemzeti Park Igazgatóság, Jósvafö.

Knollová, I., Chytrý, M., TichÝ, L., HáJeK, O., 2005: Stratified resampling of phytosociological databases: some strategies for obtaining more representative data sets for classification studies. Journal of Vegetation Science 16, 479-486.

Knollová, I., Chytrý, M., TichÝ, L., HÁJEK, O. 2006: Local ranges of phytosociological associations: are they reflected in numerical classification? Biologia (Bratislava) 61, 71-77.

Kojić, M., Mrfat-Vukelić, S., Dajić, Z., Đorđević-MilošEvić, S., 2004: Meadows and pastures of Serbia (In Serbian). Institut za istraživanja u poljoprivredi Srbije, Belgrade.

KovÁcs, J. A., 1994: Meadow associations of the Köszeg Mts. and Köszeg-hegyalja. In: Bartha, D. (ed.), Vegetation of the Köszeg Mts. (In Hungarian), 147-174. SopronKöszeg.

KovÁcs, J. A., 2002: Meadows vegetation of Örség Landscape protection area (In Hungarian). Kanitzia 10, 137-174.

LÁJER, K., 2002: Floristical and coenological studies on meadows of the Somogy county valley of the river Drava (In Hungarian). Kitaibelia 7, 187-205.

LÁJer, K., Botta-Dukát, Z., Csiky, J., Horváth, F., Szmorad, F., Bagi, I., Dobolyi, K., HAHn, I., KovÁCs, J. A., RÉDEI T., 2008: Hungarian phytosociological database (COENODATREF): sampling methodology, nomenclature and its actual stage. Annali di Botanica 7, 197-201.

LegEndRe, P., LegENdRE L., 1998: Numerical ecology. Elsevier, Amsterdam.

Lengyel, A., Chytrý, M., TichÝ L., 2011: Heterogeneity-constrained resampling of phytosociological databases. Journal of Vegetation Science 22, 175-183.

LovÁsz, Gy. (ed.), 1977: Natural geography of Baranya County (In Hungarian). Baranya Megyei Levéltár, Pécs.

Maechler, M., Rousseeuw, P., Struyf, A., Hubert M., 2005: Cluster analysis basics and extensions; unpublished.

Máthé, I., KovÁcs M., 1960: Vegetationsstudien im Mátragebirge. Acta Botanica Academiae Scientiarum Hungariae 6, 343-382.

Molnár, Zs., Bartha, S., Seregélyes, T., Illyés, E., Botta-Dukát, Z., Tímár, G., Horváth, F., Révész, A., Kun, A., Bölöni, J., Bíró, M., Bodonczi, L., DeÁK, J. Á., Fogarasi, P., Horváth, A., Isépy, I., Karas, L., Kecskés, F., Molnár, Cs., OrtMANN-NÉ AJKAI, A., RÉv, Sz., 2007: A grid-based, satellite-image supported, multi-attributed vegetation mapping method (MÉTA). Folia Geobotanica 42, 225-247.

MolnáR, Zs., BíRó, M., BölÖNI, J., HoRvÁTH, F., 2008: Distribution of the (semi-) natural habitats in Hungary. I. Marshes and grasslands. Acta Botanica Hungarica 50, 59-105.

Mucina, L., Grabherr, G., Ellmauer, T. (eds.), 1993: Die Pflanzengesellschaften Österreichs. Teil I. Anthropogene Vegetation. Gustav Fischer Verlag, Jena/Stuttgart/New York. 
Oksanen, J., Blanchet, F. G., Kindt, R., Legendre, P., O'Hara, R. G., Simpson, G. L., Sólymos, P., Stevens, M. H. H., WAGNeR, H., 2010: vegan: Community Ecology Package. R package version 1.17-0. http://cran.r-project.org/package=vegan (access: February 9,2012 )

PODANI, J. 2000: Introduction to the exploration of multivariate biological data. Backhuys Publishers, Leiden.

R Development CoRe TEAm, 2009: R: A language and environment for statistical computing. R Foundation for Statistical Computing, Vienna, Austria. http://www.R-project.org (access February 9, 2012)

Rozbrojová, Z., HÁsek, M., HÁsek, O., 2010: Vegetation diversity of mesic meadows and pastures in the West Carpathians. Preslia 87, 307-332.

SANDA, V., Popescu, A., ARCU ${ }^{\circ}$ M., 1999: Critical revision of plant communities in Romania (In Romanian). Tilia Press International, Constanța.

Soó, R., 1971: Aufzählung der Assoziationen der ungarischen Vegetation nach den neueren zönosystematisch-nomeklatorischen Ergebnissen. Acta Botanica Academiae Scientiarum Hungariae 17, 127-127.

Soó, R., 1973: Synopsis systematico-geobotanica florae vegetationisque Hungariae V. (In Hungarian) Akadémiai Kiadó, Budapest.

STANČIĆ, Z. 2000: Grasslands of class Molinio-Arrhenatheretea in northeastern Croatia (In Croatian). PhD Thesis, University of Zagreb, Zagreb.

StANČIĆ, Z., 2008: Classification of mesic and wet grasslands in northwest Croatia. Biologia (Bratislava) 63, 1089-1103.

TichÝ, L., CHYTRÝ, M., 2006: Statistical determination of diagnostic species for site groups of unequal size. Journal of Vegetation Science 17, 809-818.

TichÝ, L. 2005: New similarity indices for the assignment of relevés to the vegetation units of an existing phytosociological classification. Plant Ecology 179, 67-72.

TichÝ, L., Chytrý, M., Hásek, M., Talbot, S. S., Botta-Dukát, Z. 2010: OptimClass: Using species-to-cluster fidelity to determine the optimal partition in classification of ecological communities. Journal of Vegetation Science 21, 287-299.

TRINAJSTIĆ, I. 2002: Meadows of false oat-grass and dropworth - ass. Filipendulo vulgaris - Arrhenatheretum Hundt et Hübl. (Arrhenatherion) in Croatia (In Croatian). Agronomski Glasnik 2002, 125-136.

TrinAJSTIĆ, I. 2008: Plant communities of Republic of Croatia (In Croatian) Akademija šumarskih znanosti, Zagreb.

UhLiARovÁ, E., JaniŠOVÁ, M., UjHÁZY, K., 2007: Arrhenatherion elatioris Luquet 1926. In: JANIŠOVÁ M. (ed.), Grassland vegetation of Slovak Republic: electronic expert system for identification of syntaxa (In Slovakian), 91-109. Botanický ústav SAV, Bratislava.

van Tongeren, O., Gremmen, N., Hennekens, S., 2008: Assignment of relevés to pre-defined classes by supervised clustering of plant communities using a new composite index. Journal of Vegetation Science 19, 525-536.

ZELNIK, I., 2007: New grassland association Triseto-Centaureetum macroptili ass. nova in Slovenia. Hacquetia 6, 77-89. 
Lengyel A., Purger D., CSiky J.

ZELNIK, I., ČARNI, A., 2008: Distribution of plant communities, ecological strategy types and diversity along a moisture gradient. Community Ecology 9, 1-9.

Zuidhoff, A. C., Rodwell, J. S., Schaminée, J. H. J., 1995: The Cynosurion cristati Tx. 1947 of central, southern and western Europe: a tentative overview, based on the analysis of individual relevés. Annali di Botanica 53, 25-47. 